\title{
二甲双胍通过抑制内质网应激诱导的细胞调亡 改善结肠炎黏膜上皮屏障损伤
}

王金钢 ${ }^{1,2}$, 陈春晓 ${ }^{1}$, 任于晗 ${ }^{2}$, 周辛欣 ${ }^{1}$, 俞 珊 $^{2}$

1. 浙江大学附属第一医院消化内科, 浙江杭州 310003

2. 浙江大学附属第一医院嵊州分院嵊州市人民医院消化内科, 浙江嵊州 312400

[摘 要] 目的: 探讨二甲双胍对溃病性结肠炎秥膜上皮屏障损伤的作用及具体 机制。方法: 用人结肠癌细胞系 Caco-2 与人单核细胞系 THP-1 构建结肠炎体外细胞 共培养模型, 用 $1 \mathrm{mmol} / \mathrm{L}$ 二甲双胍作用 $24 \mathrm{~h}$ 后, 运用流式细胞术检测肠上皮细胞调 亡情况, 采用蛋白质印迹法检测紧密连接蛋白和内质网应激相关蛋白的表达水 平。结果: 与模型对照组比较, 二甲双胍组细胞调亡率从(14.22 2 2. 34)\%下降至 $(9.88 \pm 0.61) \%(t=3.119, P<0.05)$, 紧密连接蛋白 1 和密封蛋白 1 相对表达量增加 $(t=5.172$ 和 3.546 , 均 $P<0.05)$, 内质网分子伴侣葡萄糖调节蛋白 (GRP) 78 和内质网 应激诱导的调亡相关分子 $\mathrm{C} / \mathrm{EBP}$ 同源蛋白 (CHOP)、胱天蛋白酶 (caspase)-12的蛋 白表达水平下降 (均 $P<0.05$ ), 蛋白激酶 $\mathrm{R}$ 样内质网激酶 (PERK) 和真核生物起始因 子 $2 \alpha(\mathrm{eIF} 2 \alpha)$ 的磷酸化水平下降 (均 $P<0.05)$ 。结论: 二甲双胍可以通过减轻结肠炎 肠上皮细胞的细胞调亡和增加紧密连接蛋白的表达改善结肠炎肠秥膜上皮屏障损 伤, 其分子机制可能与抑制内质网应激诱导的细胞调亡途径有关。

[ 关键词] 结肠炎, 溃病性; 二甲双胍; 内质网应激; 细胞凋亡; 细胞共培养; 紧密连接 蛋白

[中图分类号 ］ R310.17 [ 文献标志码 ］ A

\section{Metformin alleviates intestinal epithelial barrier damage by inhibiting endoplasmic reticulum stress-induced cell apoptosis in colitis cell model}

WANG Jingang ${ }^{1,2}$, CHEN Chunxiao ${ }^{1}$, REN Yuhan ${ }^{2}$, ZHOU Xinxin ${ }^{1}$, YU $\operatorname{Shan}^{2}$ (1. Department of Gastroenterology, the First Affiliated Hospital, Zhejiang University School of Medicine, Hangzhou 310003, China; 2. Department of Gastroenterology, Shengzhou Branch of the First Affiliated Hospital of Zhejiang University School of Medicine, Shengzhou People's Hospital, Shengzhou 312400, Zhejiang Province, China)

收稿日期: 2021-07-10 接受日期:2021-09-20

基金项目:绍兴市2018年度科技计划(2018C30161)

第一作者:王金钢,主治医师,主要从事肠道疾病的研究;E-mail : wangjingang628@163.com; https ://orcid.org/0000-00020568-7820

通信作者:陈春晓,主任医师,硕士生导师,主要从事肠道疾病的研究;E-mail:13906523922@126.com; https://orcid.org/ 0000-0001-7536-1191 
Corresponding author: CHEN Chunxiao, E-mail: 13906523922@126.com, https:/orcid. org/0000-0001-7536-1191

\begin{abstract}
[ Abstract] Objective: To investigate the effect and mechanism of metformin on intestinal epithelial barrier injury in ulcerative colitis. Methods: A cell model of colitis was established by co-culture of human colon cancer cell line Caco-2 and human monocyte cell line THP-1. The colitis model cells were treated with metformin at concentration of $1 \mathrm{mmol} / \mathrm{L}$ for $24 \mathrm{~h}$. Flow cytometry was used to detect Caco-2 cell apoptosis, and Western blotting was used to detect the protein expression of tight junction proteins and endoplasmic reticulum stress-related proteins. Results: After metformin treatment, the apoptosis rate of Caco-2 cells was decreased from $(14.22 \pm 2.34) \%$ to $(9.88 \pm 0.61) \%(t=3.119, P<0.05)$, and the expression levels of tight junction protein-1 and claudin-1 increased $(t=5.172$ and 3.546 , both $P<0.05)$. In addition, the expression levels of endoplasmic reticulum-related proteins glucose regulated protein (GRP) 78, C/EBP homologous protein (CHOP) and caspase-12, as well as the phosphorylation level of PRKR-like endoplasmic reticulum kinase (PERK) and eukaryotic translation initiation factor $2 \alpha($ eIF $2 \alpha)$ decreased (all $P<0.05)$. Conclusion: Metformin may alleviate the intestinal epithelial barrier damage in colitis by reducing intestinal epithelial cell apoptosis and increasing the expression of tight junction proteins, which may be associated with the inhibition of endoplasmic reticulum stress-induced apoptotic pathway.
\end{abstract}

[ Key words ] Colitis, ulcerative; Metformin; Endoplasmic reticulum stress; Apoptosis; Co-culture cell; Tight junction protein

[J Zhejiang Univ(Med Sci), 2021, 50(5): 627-632.]

[缩略语] 溃疡性结肠炎 (ulcerative colitis, UC) ; 异硫氰酸荧光素(fluorescein isothiocyanate, FITC); 碘化丙啶(propidium iodide, PI); 葡萄糖调节蛋白(glucose regulated protein, GRP); 蛋白激酶 R 样内质网激酶(PRKR-like endoplasmic reticulum kinase, PERK); 真核生物起始因子 $2 \alpha$ (eukaryotic translation initiation factor $2 \alpha, \mathrm{eIF} 2 \alpha$ ); $\mathrm{C} / \mathrm{EBP}$ 同源蛋白 (C/EBP homologous protein, CHOP); 胱天蛋白酶 (cysteine aspartic acid specific protease, caspase); 聚偏二氟乙烯 (polyvinylidenefluoride,PVDF); Tris 缓冲液含 $0.1 \%$ 吐温-20 (tris-buffered saline with Tween-20,TBST); 电化学发光(electrochemiluminescence,ECL) ; 转录激活因子 (activating transcription factor, ATF)

$\mathrm{UC}$ 是一种累及直肠、结肠黏膜及黏膜下层的 慢性非特异性炎症性疾病, 主要临床表现为反复腹 痛、腹泻、黏液脓血便,是炎症性肠病的主要亚型。 $\mathrm{UC}$ 的病因和发病机制尚不清楚, 越来越多的证据 表明肠道黏膜屏障功能受损是 $\mathrm{UC}$ 发病的关键因 素 ${ }^{[1]}$ 。UC在西方发达国家的发病率和患病率很 高,近年来随着饮食、精神因素的变化,包括中国在 内的世界其他地区 $\mathrm{UC}$ 患者的数量正在迅速增 加 $^{[2]}$ 。 $\mathrm{UC}$ 患者病情多反复发作, 病程长者癌变风 险增加, 临床药物治疗远期效果也不理想 ${ }^{[3]}$, 因此
寻找 UC 的有效治疗策略具有重要意义。

肠道上皮细胞在生理状态下依赖内质网对蛋 白质进行折叠、修饰和分泌, 其在维持肠道稳态中 发挥着重要作用 ${ }^{[4]}$ 。当内质网稳态被破坏时, 错 误折叠和/或未折叠的蛋白质在内质网中积聚, 导 致细胞产生内质网应激。中等强度和短暂的内质 网应激会诱导未折叠蛋白反应, 从而减慢蛋白质 翻译速度, 导致蛋白质发生降解及折叠能力增强, 从而恢复内质网稳态; 但过强或时间过长的内质 网应激会诱导细胞发生调亡 ${ }^{[5]}$, 削弱肠道屏障功 
能和激活肠道炎症反应, 从而参与 $\mathrm{UC}$ 的发生发 展 ${ }^{[6]}$ 。因此, 靶向内质网应激是改善肠道黏膜屏 障损伤、减轻肠道炎症的潜在策略。

二甲双胍是一种在临床上广泛应用的口服抗 糖尿病药物, 除了降血糖作用外, 还具有抗炎、抗 肿瘤、抗衰老和改善代谢等作用 ${ }^{[7-10]}$ 。既往小鼠模 型和细胞模型研究均表明,二甲双胍具有增强肠 道黏膜屏障、改善肠道炎症的作用 ${ }^{[11-16]}$, 但具体机 制以及二甲双胍是否减轻内质网应激诱导的肠上 皮细胞凋亡尚不明确。本研究运用人结肠癌细胞 系 Caco-2 与人单核细胞系 THP- 1 构建结肠炎体外 细胞共培养模型, 探究二甲双胍对结肠炎肠道屏 障的作用, 并进一步探讨内质网应激及其诱导的 调亡在其中扮演的角色。

\section{1 材料与方法}

\section{1 细胞、试剂和仪器}

Caco-2 细胞和 THP-1 细胞来源于美国典型培 养物保藏中心 (ATCC)。细胞培养所用的 DMEM 高糖培养基和 RPMI 1640培养基为美国 Sigma公 司产品; 胎牛血清、胰蛋白酶、青-链霉素和非必需 氨基酸为美国 Gibco 公司产品; 二甲双胍为美国 Selleck公司产品;FITC-Annexin V/PI细胞凋亡检 测试剂盒为美国 $\mathrm{BD}$ 公司产品; 紧密连接蛋白 1 抗 体、密封蛋白 1 抗体、GRP78抗体、PERK抗体、磷 酸化eIF2 $\alpha$ 抗体、eIF2 $\alpha$ 抗体、CHOP抗体、 $\beta$-actin抗 体、细胞裂解液为美国 Cell Signaling Technology 公 司产品; 磷酸化PERK抗体、caspase-12抗体为英国 Abcam 公司产品; 上样缓冲液为上海碧云天生物 技术有限公司产品。流式细胞仪为美国 $\mathrm{BD}$ 公司 产品; 化学发光显影仪为上海勤翔科学仪器有限 公司产品。

\section{2 细胞共培养和药物干预}

Caco-2 细胞和 THP-1 细胞分别用含 $10 \%$ 胎牛 血清、1\%青-链霉素和 $1 \%$ 非必需氨基酸的 DMEM 高糖培养基和RPMI 1640 培养基培养于含 5\%二氧 化碳、 $37^{\circ} \mathrm{C}$ 培养箱中。参照文献 $[17]$ 的方法建立 结肠炎细胞模型, 即为模型对照组。二甲双胍组在 模型对照组的基础上向上室培养基中加人二甲双 胍, 使其终浓度达到 $1 \mathrm{mmol} / \mathrm{L}$, 作用时间为 $24 \mathrm{~h}$ 。

1.3 流式细胞术检测细胞凋亡情况

将模型对照组和二甲双胍组细胞用不含乙二 胺四乙酸的胰蛋白酶消化, 收集细胞, 用磷酸盐缓
冲液洗涤 2 次。向每个样本中加入 $300 \sim 400 \mu \mathrm{L}$ $1 \times$ 结合缓冲液并重悬细胞, 再加入 $5 \mu \mathrm{L}$ FITC Annexin $\mathrm{V}$ 染色液和 $5 \mu \mathrm{L}$ PI染色液, 室温避光孵育 $15 \min$ 后上机检测。

\section{4 蛋白质印迹法检测紧密连接蛋白及内质网} 应激相关蛋白的表达

弃小室内培养液, 用 $4{ }^{\circ} \mathrm{C}$ 预冷的磷酸盐缓冲 液洗涤 3 次,加人含 $1 \%$ 蛋白酶/磷酸酶抑制剂的细 胞裂解液, 冰上裂解 $30 \mathrm{~min}$, 离心后吸取蛋白上清 液用BCA 法进行蛋白定量。将提取的蛋白上清以 4: 1 的比例与 $5 \times$ 上样缓冲液混匀, $95{ }^{\circ} \mathrm{C}$ 金属浴 $10 \mathrm{~min}$ 进行蛋白变性。蛋白上样量为 $10 \sim 20 \mu \mathrm{g}$, 行 SDS-PAGE 电泳, 电泳完成后将蛋白转膜至PVDF 膜上。含 $5 \%$ 牛血清白蛋白的 TBST 溶液室温摇床 封闭 $1 \mathrm{~h}$, 一抗 $4{ }^{\circ} \mathrm{C}$ 捊育过夜。TBST 室温摇床充分 洗涤PVDF 膜 3 4 次, 每次 $10 \mathrm{~min}$ 。辣根过氧化物 酶标记二抗室温摇床孵育 $1 \mathrm{~h}$ 后, TBST洗涤PVDF 膜 3 4 次, 每次 10 min。将ECL试剂盒中的 $\mathrm{A}$ 液与 B 液按 1：1 比例混匀,并将 PVDF 膜与 ECL反应液 充分接触, PVDF 膜沥干后放人点化学发光成像仪 进行化学显影。用ImageJ 软件对蛋白条带进行灰 度值分析, 磷酸化蛋白为相对于总蛋白的表达水 平, 非磷酸化蛋白为相对于内参蛋白的表达水平。

\section{5 统计学方法}

采用GraphPad Prism 8.0 软件进行统计分 析。计量数据以均数 \pm 标准差 $(\bar{x} \pm s)$ 表示, 组间 比较采用独立样本 $t$ 检验, $P<0.05$ 为差异具有统 计学意义。

\section{2 结 果}

\section{1 二甲双胍减轻结肠炎肠上皮细胞凋亡}

模型对照组和二甲双胍组细胞调亡率分别为 (14.22 2.34$) \%$ 和 $(9.88 \pm 0.61) \%$, 差异有统计学 意义 $(t=3.119, P<0.05)$, 见图 1 。结果提示, 二甲双 胍可在一定程度上减轻结肠炎肠上皮细胞凋亡。

2.2 二甲双胍上调结肠炎细胞紧密连接蛋白的 表达

模型对照组紧密连接蛋白 1 和密封蛋白 1 相 对表达量分别为 $0.481 \pm 0.066$ 和 $0.369 \pm 0.093$, 二甲双胍组紧密连接蛋白 1 和密封蛋白 1 相对表 达量分别为 $0.775 \pm 0.092$ 和 $0.582 \pm 0.046$, 差异 有统计学意义 $(t=5.172$ 和 3.546 , 均 $P<0.05)$, 见 图 2。结果提示, 二甲双胍可上调结肠炎肠上皮细 

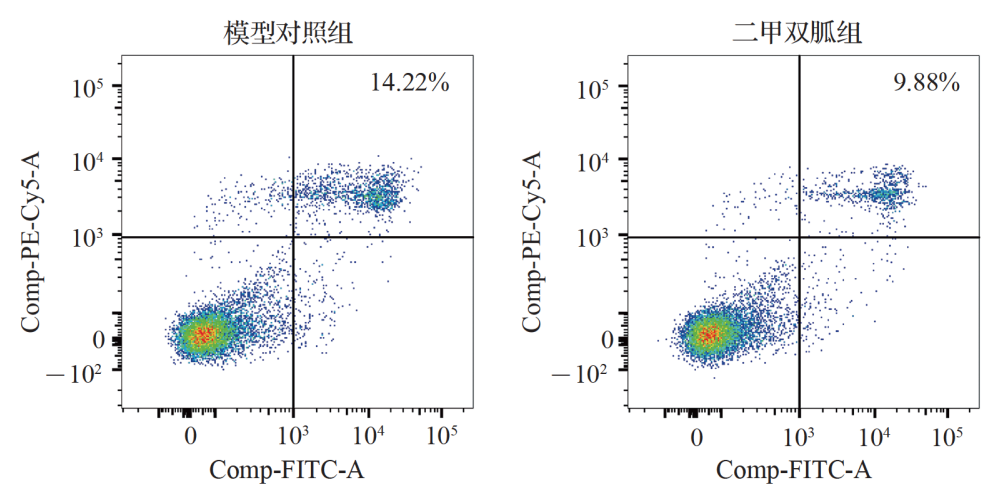

图 1 模型对照组和二甲双胍组结肠炎肠上皮细胞凋亡流式细胞图

Figure 1 The flow cytometry analysis of intestinal epithelial cell apoptosis in model group and metformin group

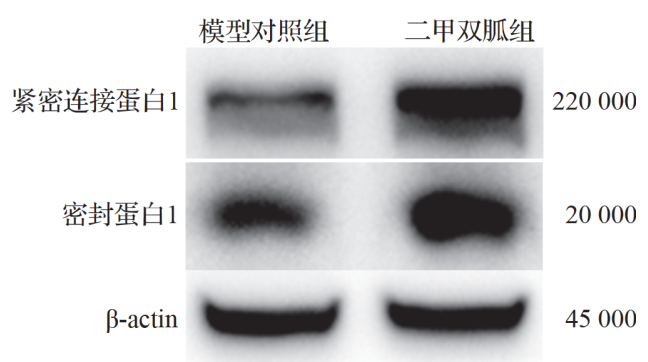

图 2 模型对照组和二甲双胍组紧密连接蛋白 1 和 密封蛋白 1 表达电泳图

Figure 2 Electrophoregrams of tight junction proteins in model group and metformin group

胞紧密连接蛋白的表达。

\section{3 二甲双胍抑制内质网应激诱导的调亡通路} 与模型对照组比较, 二甲双胍组 GRP78 和 CHOP 表达水平降低,PERK 和 $\mathrm{eIF} 2 \alpha$ 的磷酸化水 平降低 (均 $P<0.05)$, 可见 PERK-eIF $2 \alpha-A T F 4-$ CHOP 信号通路被抑制。同时, 二甲双胍组 caspase-12 表达水平也降低 $(P<0.05)$ 。见图 3 和 表 1 。结果提示, 二甲双胍可能通过抑制结肠炎肠 上皮细胞内质网应激从而减轻肠上皮细胞凋亡， 增强肠上皮屏障。

\section{3 讨 论}

针对 UC传统治疗方法存在维持缓解效果差、 激素依赖或抵抗、长期用药不良反应多等诸多问 题, 寻找有效治疗 UC 的药物是目前的研究热点。 二甲双胍作为胰岛素增敏剂, 不仅有降血糖功能, 还具有抗炎、抗衰老及降低血脂的作用 ${ }^{[7,9-10]}$ 。本 文资料显示, 二甲双胍可以减轻肠上皮细胞调亡、 增强紧密连接蛋白, 从而改善结肠炎肠道上皮屏 障损伤, 其机制可能与抑制内质网应激诱导的调
亡信号通路有关。

肠道黏膜屏障功能损伤与 $\mathrm{UC}$ 的发生发展密切相关 ${ }^{[18]}$ 。肠道黏 膜上皮屏障包括肠上皮细胞和细 胞间连接复合物 (包括紧密连接 等)。紧密连接蛋白主要由细胞膜 蛋白闭合蛋白、密封蛋白和细胞质 蛋白紧密连接蛋白 1 组成 ${ }^{[19]}$ 。UC 患者肠道上皮中紧密连接蛋白 1 、 闭合蛋白的表达水平较健康对照 者降低 ${ }^{[20]}$ 。既往在结肠炎小鼠和 细胞模型中的研究发现, 二甲双胍 能够上调紧密连接蛋白的表达, 增强肠道黏膜屏 障, 其机制可能是缘于 AMP 活化蛋白激酶信号通 路的激活 ${ }^{[11-12]}$ 。Pandey 等 ${ }^{[16]}$ 通过醋酸诱导的结 肠炎大鼠模型发现,二甲双胍可以剂量依赖性地 减轻结肠黏膜损伤,其机制可能是抑制促炎介质 的释放以及降低环氧合酶 2 、诱生型一氧化氮合酶

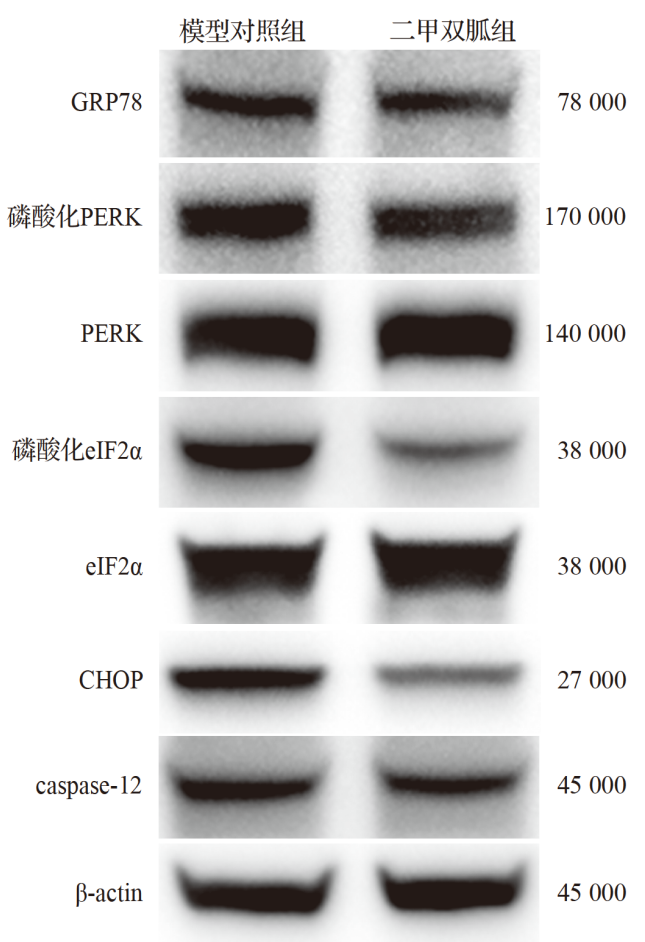

GRP : 葡萄糖调节蛋白; PERK : 蛋白激酶R样内质网激 酶; eIF $2 \alpha$ : 真核生物起始因子 $2 \alpha$; CHOP : C/EBP同源蛋白; caspase : 胱天蛋白酶.

图 3 模型对照组和二甲双胍组内质网应激相关蛋 白表达电泳图

Figure 3 Electrophoregrams of endoplasmic retculum stress associated proteins in model group and metformin group 
表 1 模型对照组和二甲双胍组内质网应激相关蛋白表达量比较

Table 1 Expression of endoplasmic reticulum stress associated proteins in model group and metformin group

\begin{tabular}{|c|c|c|c|c|c|c|}
\hline 组 别 & $n$ & GRP78 & CHOP & caspase-12 & 磷酸化PERK/PERK & 磷酸化eIF $2 \alpha / \mathrm{eIF} 2 \alpha$ \\
\hline 模型对照组 & 3 & 1. $145 \pm 0.048$ & $1.127 \pm 0.061$ & $1.173 \pm 0.076$ & $0.822 \pm 0.012$ & $0.818 \pm 0.057$ \\
\hline 二甲双胍组 & 3 & $0.925 \pm 0.138$ & $0.760 \pm 0.148$ & $0.985 \pm 0.045$ & $0.650 \pm 0.065$ & $0.568 \pm 0.126$ \\
\hline$t$ 值 & - & 3. 018 & 4.586 & 3.691 & 5.197 & 3. 618 \\
\hline$P$ 值 & - & $<0.05$ & $<0.01$ & $<0.05$ & $<0.05$ & $<0.05$ \\
\hline
\end{tabular}
核生物起始因子 $2 \alpha$.

和核因子 $\kappa \mathrm{B}$ 的表达。另有研究发现,使用二甲双 胍可降低 2 型糖尿病患者罹患炎症性肠病的风 险 ${ }^{[21]}$ 。本文资料显示, 二甲双胍可以通过减轻结 肠炎肠上皮细胞的调亡、上调紧密连接蛋白表达 水平从而增强肠道黏膜上皮屏障。

内质网应激与 $\mathrm{UC}$ 的发生发展密切相关 ${ }^{[6]}$ 。 本文资料显示, 二甲双胍作用后内质网分子伴侣 GRP78的表达下降,表明二甲双胍抑制了内质网 应激。CHOP 是 PERK 和 $\mathrm{eIF} 2 \alpha$ 磷酸化级联的下游 分子。活化的PERK 可以磷酸化 eIF $2 \alpha$,并上调 CHOP 的表达, 而 CHOP 是调节死亡受体 5 表达的 转录因子, 与内质网应激诱导的细胞调亡有 关 ${ }^{[22]}$ 。研究发现, 炎症性肠病患者肠道黏膜中 CHOP表达增强 ${ }^{[23]}$ 。caspase-12 是一种位于内质 网中的半胱氨酸蛋白酶 ${ }^{[24]}$, 可以被过强的内质网 应激激活, 再激活下游的 caspase- 9 和 caspase- 3 , 最 终导致细胞调亡 ${ }^{[25]}$ 。本文资料显示, 二甲双胍作 用后 CHOP和 caspase-12 表达水平下降, PERK 和 $\mathrm{eIF} 2 \alpha$ 的磷酸化水平降低, 表明二甲双胍抑制了内 质网应激诱导的调亡通路。以上结果提示, 二甲 双胍可能通过抑制 PERK-eIF2 $\alpha$-ATF4-CHOP信号 通路, 减轻内质网应激诱导的调亡, 从而增强结肠 炎黏膜上皮屏障。

综上所述,二甲双胍可以通过减轻肠上皮细 胞调亡和增加紧密连接蛋白表达从而改善结肠炎 肠道上皮屏障, 其分子机制可能与抑制内质网应 激诱导的细胞调亡途径有关。

\section{利益冲突 所有作者均声明不存在利益冲突}

\section{参考文献}

[1] PETERSON L W, ARTIS D. Intestinal epithelial cells: regulators of barrier function and immune homeostasis[J]. Nat Rev Immunol, 2014, 14(3):
141-153.

[2] NG S C, SHI H Y, HAMIDI N, et al. Worldwide incidence and prevalence of inflammatory bowel disease in the 21st century: a systematic review of populationbased studies $[\mathrm{J}]$. Lancet, 2017, 390(10114): 2769-2778.

[3] HIRTEN R P, SANDS B E. New therapeutics for ulcerative colitis $[J]$. Annu Rev Med, 2021, 72(1): 199-213.

[4] MA X S, DAI Z L, SUN K J, et al. Intestinal epithelial cell endoplasmic reticulum stress and inflammatory bowel disease pathogenesis: an update $\operatorname{review}[\mathrm{J}]$. Front Immunol, 2017, 8: 1271.

[5] EUGENE S P, REDDY V S, TRINATH J. Endoplasmic reticulum stress and intestinal inflammation: a perilous union[J]. Front Immunol, 2020, 11: 543022.

[6] KASER A, BLUMBERG R S. Endoplasmic reticulum stress in the intestinal epithelium and inflammatory bowel disease $[J]$. Semin Immunol, 2009, 21(3): 156-163.

[7] BAI B, CHEN H B. Metformin: a novel weapon against inflammation $[\mathrm{J}]$. Front Pharmacol, 2021, 12: 622262.

[8] CHEN Y C, LI H, WANG J. Mechanisms of metformin inhibiting cancer invasion and migration $[\mathrm{J}]$. Am J Transl Res, 2020, 12(9): 4885-4901.

[9] SOUKAS A A, HAO H, WU L. Metformin as anti-aging therapy: is it for everyone?[J]. Trends Endocrinol Metab, 2019, 30(10): 745-755.

[10] HE L. Metformin and systemic metabolism[J]. Trends Pharmacol Sci, 2020, 41(11): 868-881.

[11] CHEN L, WANG J, YOU Q, et al. Activating AMPK to restore tight junction assembly in intestinal epithelium and to attenuate experimental colitis by metformin $[\mathrm{J}]$. Front Pharmacol, 2018, 9: 761.

[12] DENG J, ZENG L, LAI X, et al. Metformin protects against intestinal barrier dysfunction via AMPK $\alpha 1$ dependent inhibition of JNK signalling activation $[\mathrm{J}]$. J Cell Mol Med, 2018, 22(1): 546-557.

[13 ] DI FUSCO D, DINALLO V, MONTELEONE I, et al. Metformin inhibits inflammatory signals in the gut by 
controlling AMPK and p38 MAP kinase activation $[\mathrm{J}]$. Clin Sci, 2018, 132(11): 1155-1168.

[14] EL-MAHDY N A, EL-SAYAD M E S, EL-KADEM A $\mathrm{H}$, et al. Metformin alleviates inflammation in oxazolone induced ulcerative colitis in rats: plausible role of sphingosine kinase 1/sphingosine 1 phosphate signaling pathway $[\mathrm{J}]$. Immunopharmacol Immunotoxicol, 2021, 43(2): 192-202.

[15] LIU X, SUN Z, WANG H. Metformin alleviates experimental colitis in mice by up-regulating TGF- $\beta$ signaling $[\mathrm{J}]$. Biotech Histochem, 2021, 96(2): 146-152.

[16] PANDEY A, VERMA S, KUMAR V L. Metformin maintains mucosal integrity in experimental model of colitis by inhibiting oxidative stress and proinflammatory signaling $[\mathrm{J}]$. Biomed Pharmacother, 2017, 94: 1121-1128.

[17] REN M T, GU M L, ZHOU X X, et al. Sirtuin 1 alleviates endoplasmic reticulum stress-mediated apoptosis of intestinal epithelial cells in ulcerative colitis $[\mathrm{J}]$. World J Gastroenterol, 2019, 25(38): 5800-5813.

[18] VAN DER POST S, JABBAR K S, BIRCHENOUGH $\mathrm{G}$, et al. Structural weakening of the colonic mucus barrier is an early event in ulcerative colitis pathogenesis[J]. Gut, 2019, 68(12): 2142-2151.

[19] PARADIS T, BÈGUE H, BASMACIYAN L, et al. Tight junctions as a key for pathogens invasion in intestinal epithelial cells $[$ J $]$. Int J Mol Sci, 2021, 22(5): 2506.

[20] TAN Y, GUAN Y, SUN Y, et al. Correlation of intestinal mucosal healing and tight junction protein expression in ulcerative colitis patients $[\mathbf{J}]$. Am J Med Sci, 2019, 357(3): 195-204.

[21] TSENG C H. Metformin use is associated with a lower risk of inflammatory bowel disease in patients with type 2 diabetes mellitus[J]. J Crohns Colitis, 2021, 15(1): 64-73.

[22] LU M, LAWRENCE D A, MARSTERS S, et al. Opposing unfolded-protein-response signals converge on death receptor 5 to control apoptosis $[J]$. Science, 2014, 345(6192): 98-101.

[23 ] KASER A, LEE A H, FRANKE A, et al. XBP1 links ER stress to intestinal inflammation and confers genetic risk for human inflammatory bowel disease[J]. Cell, 2008, 134(5): 743-756.

[24] YONEDA T, IMAIZUMI K, OONO K, et al. Activation of caspase-12, an endoplastic reticulum (ER) resident caspase, through tumor necrosis factor receptorassociated factor 2-dependent mechanism in response to the ER stress[J]. J Biol Chem, 2001, 276(17): 13935-13940.

[25] IURLARO R, MUÑOZ-PINEDO C. Cell death induced by endoplasmic reticulum stress[J]. FEBS J, 2016, 283(14): 2640-2652.

[本文编辑 沈 敏 余 方]

\section{高建青教授团队构建氧化铁纳米粒增强的干细胞载体系统 实现脑胶质瘤的高效靶向自杀基因治疗}

2021年7月13日，《治疗诊断学》(Theranostics) 在线发表了浙江大学药学院高建青教授团队的最新研究成果 “Iron oxide nanoparticles promote Cx43-overexpression of mesenchymal stem cells for efficient suicide gene therapy during glioma treatment” (https://doi. org/10.7150/thno. 60160)。该研究成功制备了一种基于氧化铁纳米粒增强的间充质干细胞 (MSCs)载体系统, 不但实现了自杀基因向脑胶质瘤组织的高效靶向递送,还能通过氧化铁纳米粒增强MSCs 与胶质瘤细 胞间的间隙连接通信,显著提高胶质瘤细胞对旁观者杀伤效应的敏感性,最终实现良好的抑瘤效果。

研究人员针对当前基于MSCs的细胞载体系统用于脑胶质瘤治疗的不足,通过氧化铁纳米粒携载自杀基因对MSCs 载体进行改良,最终实现了MSCs 载体对自杀基因(疮疹病毒胸苷激酶)的高效携载和向胶质瘤组织的靶向递送。研究进 一步发现,氧化铁纳米粒能上调MSCs的细胞间隙连接蛋白 43 表达,并可以进一步影响与MSCs接触的胶质瘤细胞上的该 蛋白表达,促进MSCs与胶质瘤细胞间的间隙连接通信,提高自杀基因疗法旁观者效应的杀伤效率。

李艾硕士研究生和张添源博士为论文第一作者。该研究得到了国家自然科学基金、中国博士后科学基金以及浙江 省 “万人计划” 等资助。 